\section{NUCLEAR PHYSICS}

\section{Shape shifters}

Phys. Rev. Lett. (in the press); preprint at https://arxiv.org/abs/1606.09056

Electron dynamics usually steal the show when we talk about atoms. But that doesn't mean that atomic nuclei are simply static. The collective motion of nucleons is what creates a particular nuclear shape and understanding how this works can help us to manipulate exotic nuclei. So what about isotopes? Their nuclei always host the same number of protons, but when we start adding neutrons, things can get pretty crowded - leading to nuclear shape deformation.

Typically, increasing the number of neutrons leads to a gradual change in the nuclear shape, for instance from spherical to prolate or oblate. But Tomoaki Togashi and colleagues have now established that this is not the case for zirconium, whose nuclear shape undergoes an abrupt deformation at around 60 neutrons. By performing systematic large-scale Monte Carlo shellmodel calculations, the authors were able to reproduce this sudden nuclear reorganization - and demonstrate that it behaves as a quantum phase transition.

\section{LASER-PLASMA ACCELERATION}

\section{Surf's up}

Nat. Commun. 7, 12891 (2016)

When a plasma interacts with a high-intensity laser pulse, the resulting collective motion of the electrons in the plasma can generate an accelerating electric field, providing conditions for the electrons to rapidly 'surf' away. Based on this insight, a number of different laser-plasma particle acceleration schemes have been proposed.
Bruno Gonzalez-Izquierdo and colleagues have looked at the plasma that is formed when a high-intensity femtosecond laser pulse is incident on a 10 -nm-thick aluminium target coated with hydrocarbon layers. The onset of the pulse locally generates a plasma in the aluminium foil, which then serves as an aperture for the remainder of the pulse, causing it to diffract. The diffracted beam leads to an accelerating electric field, in this case driving away protons, which are freed from the hydrocarbon coating during irradiation.

To assess the potential of this scheme as a compact proton accelerator, the authors varied the polarization of the laser light linear, elliptic or circular - and found that it significantly affected the diffractive behaviour of the aperture, and ultimately the proton beam's intensity profile. Tuning the polarization's ellipticity resulted in different distributions, featuring ring, bubble or striped features.

\section{EVAPORATION}

\section{Worth all the sweat}

Proc. Natl Acad. Sci. USA 113, 10275-10280 (2016)

FL Our skin saves us. Without it, the cells in our bodies would quickly dry out. Beyond presenting a physical barrier, mammalian skin also actively keeps the water-evaporation rate practically constant, up to a relative humidity of $\sim 85 \%$. Kevin Roger and colleagues have now found that simple aqueous solutions of amphiphilic molecules - which have both hydrophilic and hydrophobic parts - mimic this sort of humidity buffering.

Using optical and infrared microscopy as well as small-angle $\mathrm{X}$-ray scattering, they found that the key to the skin-like behaviour in these solutions is an air-liquid interface that

\title{
INTRACELLULAR TRANSPORT
}

\section{With a little help from my friends}

In the messy world of the cell, vesicles are the preferred vehicles for intracellular transport.

These fluid-filled membrane structures package cargo up and overcome the logistical nightmare of locating, binding and fusing with a target membrane to deliver their goods. But they aren't in it alone. Long, rod-like tethering molecules are tasked with capturing incoming vesicles, then bringing them in to dock. How they achieve this is not fully understood, but the answer might lie in a simple physical mechanism, as David H. Murray and colleagues have now determined.

Using optical tweezers on tethering machinery built from scratch, they found that tether length ranged from the predicted $200 \mathrm{~nm}$, all the way down to an order of magnitude shorter - motivating a hypothesis that a conformational change facilitates docking. They fitted electron microscopy data with the worm-like chain model to determine that the protein charged with regulating recruitment of tethering molecules actually altered their persistence length. The increased flexibility made the out-of-equilibrium conformation of the extended tether vulnerable to entropic collapse, which the authors were able to measure, demonstrating that the tether could function to both capture the vesicle and reel it in. reacts through self-assembly to changes in humidity. This process controls the thickness and structure of the interface - and thus its permeability - resulting in a feedback loop where stronger driving forces due to increasing gradients in relative humidity are balanced by decreasing the permeability of the interface.

A similar sort of feedback loop could be at work in the stratum corneum layer of our skin, where phase transitions in the lipid self-assembly structure have been observed. Having a fuller understanding of the underlying mechanism might lead to fresh approaches to remedy skin diseases.

AHT

\section{BLACK HOLES}

\section{The dark between the stars}

Mon. Not. R. Astron. Soc. 462, 2333-2342 (2016)

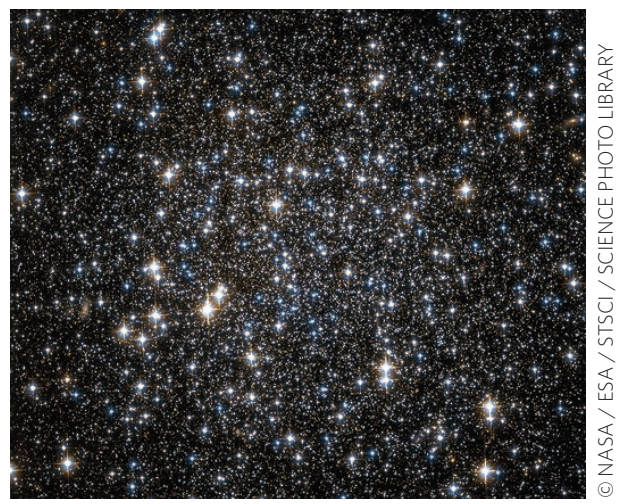

Globular clusters are spherical star formations gravitating around galactic cores. During their evolution, lighter stars tend to move away from the centre, which is left populated largely with stars of higher mass. But globular cluster NGC 6101 (pictured) is peculiar. Its stars are just too evenly distributed - devoid of the mass segregation expected for its size and age. Using extensive simulations of the dynamic evolution of the cluster over thirteen billion years, Miklos Peuten and colleagues were able to figure out the reason why: the lack of segregation is due to the existence of hundreds of black holes.

So where do these black holes come from? We know that they are the remnants of explosions of massive stars, but current theories predict that these black holes would be kicked away from their parent star. The simulations of Peuten et al. account for the current star distribution in NGC 6101 , including hundreds of black holes of stellar mass. But they also suggest that the kick black holes receive after birth is lower than expected.

Written by lulia Georgescu, Abigail Klopper, Federico Levi, Andreas H. Trabesinger and Bart Verberck. 